\title{
Higher order net baryon number cumulants in the strong coupling lattice QCD *
}

\author{
Terukazu Ichihara \\ Department of Physics \& Yukawa Institute for Theoretical Physics, \\ Kyoto University, Kyoto 606-8502, Japan \\ E-mail: t-ichieruby.scphys.kyoto-u.ac.jp
}

\author{
Akira Ohnishi \\ Yukawa Institute for Theoretical Physics, \\ Kyoto University, Kyoto 606-8502, Japan \\ E-mail: ohnishidyukawa.kyoto-u.ac.jp

\section{Kenji Morita} \\ Yukawa Institute for Theoretical Physics, \\ Kyoto University, Kyoto 606-8502, Japan \\ Institute of Theoretical Physics, \\ University of Wroclaw, PL-50204 Wroctaw, Poland \\ E-mail: kmoritadyukawa.kyoto-u.ac.jp
}

\begin{abstract}
We study the net-baryon number cumulant ratios, $S \sigma$ and $\kappa \sigma^{2}$, at finite temperature and baryon chemical potential in the strong coupling and chiral limits of the lattice QCD. We find nonmonotonic behaviors of the cumulant ratios as functions of $T$ in the vicinity of the phase boundary at $\mu / T=0.2$ and 0.8 , as expected from the $O(4)$ scaling function analysis. In particular, we also find that there is a region where the cumulant ratios become negative when approaching to the expected tricritical point probably due to the finite size effect.
\end{abstract}

The 33rd International Symposium on Lattice Field Theory

14 - 18 July 2015

Kobe International Conference Center, Kobe, Japan

*Report No. : YITP-15-92, KUNS-2591

† Speaker. 


\section{Introduction}

Phase transitions in Quantum Chromodynamics (QCD) at finite baryon density have been of great interest both from theoretical and experimental point of view. In the beam energy scan program at Relativistic Heavy Ion collider (RHIC), higher order cumulants of event-by-event netproton number multiplicity distribution have been measured as a promising observable of the possible critical phenomena associated with the phase transition in QCD. Indeed, recent experimental data on cumulants for high multiplicity events show non-monotonic behavior as a function of the colliding energy. The values of the cumulants deviate from a non-critical baseline given by the Skellam distribution [1], which corresponds to a distribution for the particle minus anti-particle each of which follows the Poisson distribution.

One of the theoretical arguments relevant for the non-monotonic behavior of the net-proton number cumulants is the influence of the QCD critical point [2]. Around the critical point, the kurtosis can be negative due to the three dimensional Z(2) criticality which is the expected universality class for the QCD critical point [3]. Alternatively, the three-dimensional $\mathrm{O}(4)$ criticality around the chiral phase transition can also lead to the anomalous behavior in the cumulants [4]. Since the singular part of the kurtosis positively diverge from and above the critical temperature in the chiral limit $\left(m_{0} \rightarrow 0\right)$, it has one negative peak between two positive peaks at finite quark mass. The singular part of the second order cumulant $\left(\sigma^{2}\right)$ positively diverges at the critical temperature in the chiral limit $\left(m_{0} \rightarrow 0\right)$. At finite quark mass, the divergence is smeared and $\sigma^{2}$ has a positive peak at the pseudo-critical temperature. Then the fourth-order cumulant $\left(\kappa \sigma^{4}\right)$ should have a negative region between the inflection points of $\sigma^{2}$, since $\kappa \sigma^{4}$ is equivalent to the second derivative of $\sigma^{2}$ with respect to $\hat{\mu}=N_{c} \mu / T$. These arguments are deduced from the universality arguments for the singular part of the pressure based on the relevant symmetry breaking pattern of the phase transition in the thermodynamic limit. To interpret the experimental data, we need explicit calculations to see how much criticality realizes in realistic cases including the regular part and finite volume effects.

While so far such calculations have been carried out mainly by using chiral models, there are four points which one needs to improve toward the results for QCD at physical points. First, we need a theoretical framework with fluctuation effects beyond the mean field treatments, since the critical behavior is governed by fluctuations of soft modes which gives critical exponents different from the mean field values. Second, it is desirable to investigate the finite chemical potential effects non-perturbatively, while it is generally difficult in the lattice QCD (LQCD). The third one is that we should make calculations at light quark mass around the physical point, and hopefully we should compare the results with those in the chiral limit, to understand the remnant of the $Z(2)$ or $O(4)$ criticality. The fourth one is the finite volume effects. The thermodynamic and continuum limit results should be compared with the finite volume results.

Strong coupling lattice QCD (SC-LQCD) satisfies three of the above four points except that we cannot take the continuum limit [5-12]. SC-LQCD provides an approximate framework starting from the lattice QCD action, assuming that the coupling constant is sufficiently large; we expand the partition function in $1 / g^{2}$ by evaluating the plaquette term $\left(\propto 1 / g^{2}\right)$ effects perturbatively. While the lattice spacing is coarse and we can describe only the confined objects in SC-LQCD, it can describe the chiral phase transition by using the strong coupling action with fermions even in 
the chiral limit. Recently, it has been demonstrated that we can take account of fluctuation effects in SC-LQCD by using the monomer-dimer-polymer (MDP) simulation [13-15] and auxiliary field Monte Carlo (AFMC) method [16]. In the strong coupling limit, the sign problem is so mild that we could study the chiral phase transition with fermions $[14,16]$. By integrating out the (spatial) link variables first in MDP (AFMC), we obtain the effective action composed of color singlet quark composites. States specified by color singlet auxiliary fields or MDP loop configurations would be closer to eigen states of QCD, then the complex phases could be suppressed.

In this work, we utilize one species of unrooted staggered fermion, which corresponds to $N_{f}=4$ in the continuum limit but has $\mathrm{O}(2)$ symmetry at finite lattice spacing. Since the sign of the relevant critical exponent in $\mathrm{O}(2)$ is the same as that in $\mathrm{O}(4)$ realized in the massless two-flavor case, we could anticipate that the higher-order cumulant ratios show qualitatively similar behavior in $\mathrm{O}(2)$ and $\mathrm{O}(4)$ cases. We perform Monte Carlo simulations for auxiliary fields to take account of mesonic fluctuation effects in the chiral limit. In this proceedings, we will show numerical results of higher order cumulant ratios of the net-baryon number at finite temperature and chemical potential to $8^{4}$ lattice in the chiral limit.

\section{Effective action of strong coupling lattice QCD in Auxiliary field Monte Carlo method in the strong coupling limit}

In this section, we introduce the auxiliary field effective action in the strong coupling limit developed in Ref. [16]. We consider here a lattice QCD action for color $\mathrm{SU}\left(N_{c}=3\right)$ with one species of unrooted staggered fermion, that has $\mathrm{O}(2)$ symmetry at finite lattice spacing, on an anisotropic lattice in $d+1$ dimensional $(d=3)$ spacetime. Temporal and spatial lattice sizes are denoted as $N_{\tau}$ and $L$, respectively. We set the lattice spacing as $a=1$ throughout this paper. In the strong coupling limit, we could ignore the plaquette terms. The effective action is obtained in the strong coupling limit and in the leading order of the large dimensional expansion [6] by integrating out spatial-link variables as [7-12]

$$
\begin{aligned}
S_{\mathrm{eff}} & =\frac{1}{2} \sum_{x}\left[V_{x}^{+}-V_{x}^{-}\right]-\frac{1}{4 N_{c}} \sum_{x, j} M_{x} M_{x+\hat{j}}+m_{0} \sum_{x} M_{x}, \\
M_{x} & =\bar{\chi}_{x} \chi_{x}, \quad V_{x}^{+}=\gamma e^{\mu / f(\gamma)} \bar{\chi}_{x} U_{0, x} \chi_{x+\hat{0}}, \quad V_{x}^{-}=\gamma e^{-\mu / f(\gamma)} \bar{\chi}_{x+\hat{0}} U_{0, x}^{\dagger} \chi_{x},
\end{aligned}
$$

where $\chi_{x}$ is the quark field and the mesonic composites are represented as $M_{x}, V_{x}^{+}$, and $V_{x}^{-} \cdot \mu$ is the quark chemical potential and $f(\gamma)=a / a_{\tau}$ is the ratio of temporal and spatial physical lattice spacing. We adopt a simple parameterization $f(\gamma)=\gamma^{2}$ in SCL determined from the quantum correction under the mean field approximation [8]. As a result, temperature can be written as $T=\gamma^{2} / N_{\tau}[8]$.

We apply the extended Hubbard-Stratonovich transformation [11,12] to four-Fermi terms $M_{x} M_{x+\hat{j}}$ in Eq. (لـ (ل) The four-Fermi terms are semi-diagonal in the momentum representation, and there exist positive $\left(f(\mathbf{k})=\sum_{j=1}^{d} \cos k_{j}>0\right)$ and negative $(f(\mathbf{k})<0)$ modes,

$$
-\frac{1}{4 N_{c}} \sum_{x, j} M_{x} M_{x+\hat{j}}=-\frac{L^{3}}{4 N_{c}} \sum_{\mathbf{k}, \tau, f(\mathbf{k})>0} f(\mathbf{k})\left(M_{\mathbf{k}, \tau} M_{-\mathbf{k}, \tau}-M_{\overline{\mathbf{k}}, \tau} M_{-\overline{\mathbf{k}}, \tau}\right),
$$


where $M_{x=(\mathbf{x}, \tau)}=\sum_{\mathbf{k}} e^{i \mathbf{k} \cdot \mathbf{x}} M_{\mathbf{k}, \tau}, \overline{\mathbf{k}}=\mathbf{k}+(\pi, \pi, \pi)$, and $f(\overline{\mathbf{k}})=-f(\mathbf{k})$. Bosonization of negative modes involves with $i \varepsilon_{x}\left(\varepsilon_{x}=(-1)^{x_{0}+x_{1}+x_{2}+x_{3}}\right)$, and the effective action after the bosonization is obtained as

$$
\begin{aligned}
S_{\mathrm{eff}}^{\mathrm{EHS}} & =\frac{1}{2} \sum_{x}\left[V_{x}^{+}-V_{x}^{-}\right]+\sum_{x} m_{x} M_{x}+\frac{L^{3}}{4 N_{c}} \sum_{\mathbf{k}, \tau, f(\mathbf{k})>0} f(\mathbf{k})\left[\left|\sigma_{\mathbf{k}, \tau}\right|^{2}+\left|\pi_{\mathbf{k}, \tau}\right|^{2}\right] \\
m_{x} & =m_{0}+\frac{1}{4 N_{c}} \sum_{j}\left[(\sigma+i \varepsilon \pi)_{x+\hat{j}}+(\sigma+i \varepsilon \pi)_{x-\hat{j}}\right]
\end{aligned}
$$

where $\sigma_{x}=\sum_{\mathbf{k}, f(\mathbf{k})>0} e^{i \mathbf{k} \cdot \mathbf{x}} \sigma_{\mathbf{k}, \tau}$ and $\pi_{x}=\sum_{\mathbf{k}, f(\mathbf{k})>0}(-1)^{\tau} e^{i \mathbf{k} \cdot \mathbf{x}} \pi_{\mathbf{k}, \tau}$. It should be noted that $\mathbf{k}$ and $-\mathbf{k}$ fields are related via the real field conditions, $\sigma_{-\mathbf{k}, \tau}=\sigma_{\mathbf{k}, \tau}^{*}$ and $\pi_{-\mathbf{k}, \tau}=\pi_{\mathbf{k}, \tau}^{*}$. In this bosonization procedure, we introduce auxiliary fields to preserve original $\mathrm{O}(2)$ symmetry $[16,17]$.

The partition function and the effective action are obtained after integrating over the Grassmann and temporal link $\left(U_{0}\right)$ variables as $[7,8]$,

$$
\begin{aligned}
\mathscr{Z}_{\mathrm{AF}} & =\int \mathscr{D}\left[\sigma_{\mathbf{k}, \tau}, \pi_{\mathbf{k}, \tau}\right] e^{-S_{\mathrm{eff}}^{\mathrm{AF}}}, \\
S_{\mathrm{eff}}^{\mathrm{AF}} & =\sum_{\mathbf{k}, \tau, f(\mathbf{k})>0} \frac{L^{3} f(\mathbf{k})}{4 N_{c}}\left[\left|\sigma_{\mathbf{k}, \tau}\right|^{2}+\left|\pi_{\mathbf{k}, \tau}\right|^{2}\right]-\sum_{\mathbf{x}} \log R(\mathbf{x}), \\
R(\mathbf{x}) & =X_{N_{\tau}}(\mathbf{x})^{3}-2 X_{N_{\tau}}(\mathbf{x})+2 \cosh \left(N_{c} \mu / T\right),
\end{aligned}
$$

where $\mathscr{D}\left[\sigma_{\mathbf{k}, \tau}, \pi_{\mathbf{k}, \tau}\right]=\prod_{\mathbf{k}, \tau, f(\mathbf{k})>0} d \sigma_{\mathbf{k}, \tau} d \sigma_{\mathbf{k}, \tau}^{*} d \pi_{\mathbf{k}, \tau} d \pi_{\mathbf{k}, \tau}^{*} . X_{N_{\tau}}(\mathbf{x})$ is calculated by using a recursion formula as in Refs. [7,8]. In the static case, we find $X_{N_{\tau}}=2 \cosh \left(N_{\tau} \operatorname{arcsinh}\left(m_{x} / \gamma\right)\right)$.

In order to take account of mesonic fluctuation effects, we integrate over mesonic auxiliary fields $\left(\sigma_{\mathbf{k}, \tau}, \pi_{\mathbf{k}, \tau}\right)$ by using the Monte Carlo method with the effective action $S_{\mathrm{eff}}^{\mathrm{AF}}$. By virtue of the milder sign problem than the standard LQCD, we can perform Monte Carlo simulations at both finite $T$ and $\mu$. We use the the reweighting method at finite $\mu$ and $T$, and calculate observables.

\section{Higher-order cumulant ratios with mesonic fluctuation effects for net-baryon number}

We shall now discuss higher-order cumulant ratios of the net-baryon number obtained in the chiral limit $\left(m_{0} \rightarrow 0\right)$ on $4^{3} \times 4,6^{3} \times 4,6^{3} \times 6$ and $8^{3} \times 8$ lattices. The results except for the $8^{3} \times 8$ lattice are reported in Ref. [17]. The normalized skewness $S \sigma$ and kurtosis $\kappa \sigma^{2}$ are defined as

$$
S \sigma=\frac{\chi_{\mu}^{(3)}}{\chi_{\mu}^{(2)}}, \kappa \sigma^{2}=\frac{\chi_{\mu}^{(4)}}{\chi_{\mu}^{(2)}}, \chi_{\mu}^{(n)}=\frac{1}{V T^{3}} \frac{\partial^{n} \log Z}{\partial \hat{\mu}^{n}},
$$

where $\hat{\mu}=N_{c} \mu / T$ [18]. $S, \kappa$, and $\sigma^{2}$ are skewness, kurtosis, and variance, respectively.

We first discuss the results at $\mu / T=0.2$. In the present framework, the tricritical point (TCP) exists between $\mu / T=0.8$ and 1.0 in the strong coupling limit [16], and $\mu / T=0.2$ is away from TCP. In Fig. 四, we show the normalized skewness $(S \sigma)$ and kurtosis $\left(\kappa \sigma^{2}\right)$ at $\mu / T=0.2$ as functions of $T / T_{c}$, where $T_{c} \simeq 1.44$ is the critical temperature at $\mu=0$ evaluated on a $8^{4}$ lattice. The skewness has one positive peak, whose height grows with increasing lattice size. The kurtosis also 
$\mathrm{S} \sigma, \mu / \mathrm{T}=0.2$

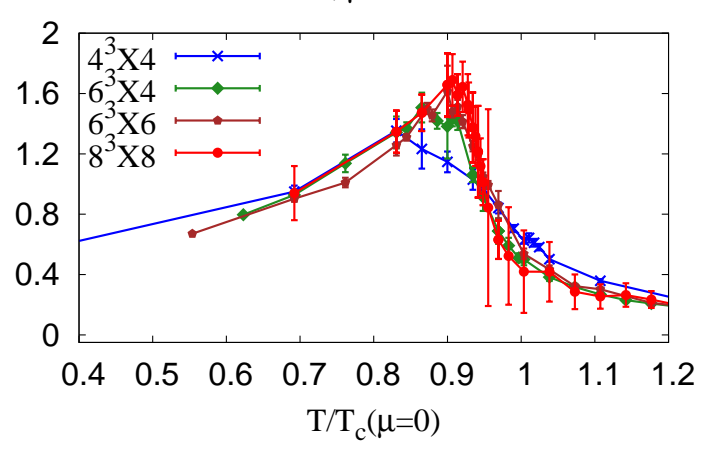

$\kappa \sigma^{2}, \mu / \mathrm{T}=0.2$

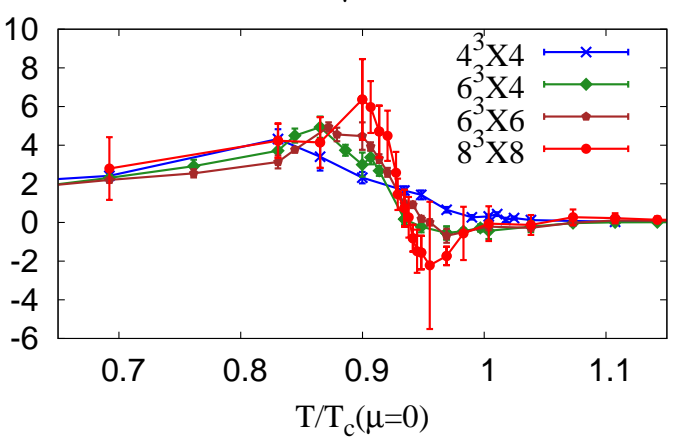

Figure 1: The cumulant ratios $S \sigma$ and $\kappa \sigma^{2}$ on the $\mu / T=0.2$ line as functions of $T / T_{c}(\mu=0)$.

has one positive peak. On larger lattices, we find a negative kurtosis region, which might originate from the phase transition nature smeared by finite size effects [19]. The singular contribution of the kurtosis may overcome the regular part of the kurtosis with increasing lattice size [4].

Next, let us examine the behavior of cumulants around TCP. In Fig. \, we show the normalized skewness and kurtosis divided by the spatial volume, $S \sigma / L^{3}$ and $\kappa \sigma^{2} / L^{3}$, at $\mu / T=0.8$. We find significant non-monotonic behavior around the phase boundary. The skewness has one positive peak and one negative valley, and the kurtosis has two positive peaks and one negative valley in between. With increasing lattice size, the amplitudes of $S \sigma$ and $\kappa \sigma^{2}$ increase, and the peaks and valley become narrower. These features are consistent with the expectations from the $\mathrm{O}(4)$ scaling analysis in the thermodynamic and chiral limits; $S \sigma$ diverges positively (negatively) toward the phase transition temperature from below (above), and $\kappa \sigma^{2}$ diverges positively at the phase transition temperature [4]. The negative region of $\kappa \sigma^{2}$ presumably appears due to the smeared singular part, as observed with finite quark mass in the thermodynamic limit [4]. While the smearing from the finite mass and the finite size may be different, the present results imply that the finite size masks the singular behavior [19] in a similar way to the finite mass.

\section{Summary}

We have studied the cumulant ratios, $S \sigma$ and $\kappa \sigma^{2}$, of the net-baryon number at finite $T$ and $\mu$ on the lattice in the strong coupling and chiral limits by using the auxiliary field Monte Carlo method. We find non-monotonic behavior of $S \sigma$ and $\kappa \sigma^{2}$ at around the phase boundary near the expected TCP.

The normalized skewness $S \sigma$ has one positive peak at $\mu / T=0.2$ and has one positive and one negative peaks at $\mu / T=0.8$. The peak width at $\mu / T=0.8$ shrinks with increasing lattice size. The normalized kurtosis $\kappa \sigma^{2}$ has one positive peak and one negative peak on larger lattices at $\mu / T=0.2$, and two positive peaks and one negative valley at $\mu / T=0.8$. The valley width at $\mu / T=0.8$ shrinks with larger lattice size, then we could expect that the normalized kurtosis 

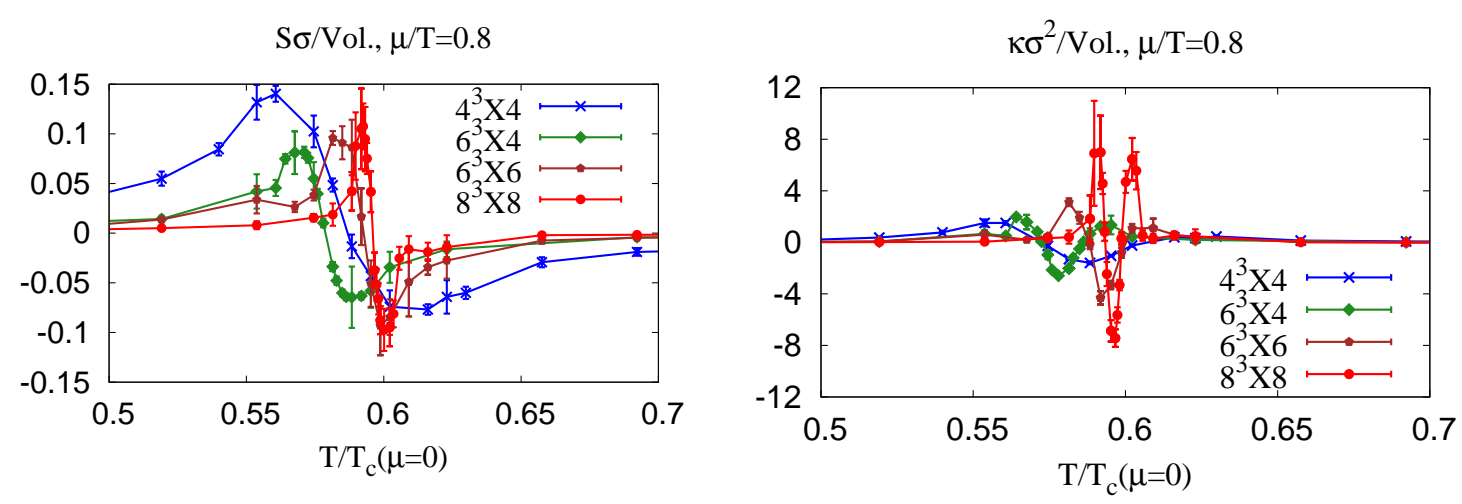

Figure 2: The cumulant ratios $S \sigma$ and $\kappa \sigma^{2}$ divided by the space volume on the $\mu / T=0.8$ line as functions of $T / T_{c}(\mu=0)$.

should positively diverge in the thermodynamic and chiral limits as observed in the to $O(4)$ scaling analysis [4].

Lattice-size dependence on $S \sigma$ and $\kappa \sigma^{2}$ implies that the finite size masks the singular behavior of these cumulant ratios as in the finite mass cases [19]. While the finite size and finite mass effects are different, we may conclude that the negative region of $\kappa \sigma^{2}$ appears due to the finite size effect in the chiral limit $[4,19]$.

\section{Acknowledgments}

The authors would like to thank Sinya Aoki, Frithjof Karsch, Swagato Mukherjee, Hiroshi Ohno, Philippe de Forcrand, Hideaki Iida, Yu Maezawa, Keitaro Nagata, Shuntaro Sakai, Takahiro Sasaki, and Wolfgang Unger and participants of the 33rd Int. Symp. on Lattice Field Theory (Lattice 2015) for useful discussions. Part of the numerical computation in this work was carried out at the Yukawa Institute Computer Facility. TI is supported by the Grants-in-Aid for the Japan Society for the Promotion of Science (JSPS) Fellows (No. 25-2059) and would like to thank the RIKEN-BNL Brain Circulation Program. KM was supported by the Polish Science Foundation (NCN), under Maestro grant 2013/10/A/ST2/00106. This work is supported in part by KAKENHI (Nos. 23340067, 24340054, 24540271, 15K05079, 24105001, 24105008), and by the Yukawa International Program for Quark-hadron Sciences.

\section{References}

[1] M. M. Aggarwal et al. (STAR Collaboration), Phys. Rev. Lett. 105, 022302 (2010); L. Adamczyk, et al. (STAR Collaboration), Phys. Rev. Lett. 112, 032302 (2014).

[2] M. A. Stephanov, Phys. Rev. Lett. 102, 032301 (2009).

[3] M. A. Stephanov, Phys. Rev. Lett. 107, 052301 (2011) [arXiv:1104.1627 [hep-ph]].

[4] B. Friman, F. Karsch, K. Redlich, and V. Skokov, Eur. Phys. J. C 71, 1694 (2011). 
[5] N. Kawamoto and J. Smit, Nucl. Phys. B192, 100 (1981); H. Kluberg-Stern, A. Morel, and B. Petersson, Phys. Lett. B 114, 152 (1982); J. Hoek, N. Kawamoto, and J. Smit, Nucl. Phys. B 199, 495 (1982); P. H. Damgaard, N. Kawamoto and K. Shigemoto, Phys. Rev. Lett. 53, 2211 (1984); P. H. Damgaard, D. Hochberg, and N. Kawamoto, Phys. Lett. B 158, 239 (1985); P. H. Damgaard, N. Kawamoto, and K. Shigemoto, Nucl. Phys. B 264, 1 (1986); V. Azcoiti, G. Di Carlo, A. Galante, and V. Laliena, J. High Energy Phys. 09, 014 (2003); K. Fukushima, Prog. Theor. Phys. Suppl. 153, 204 (2004) [hep-ph/0312057]; Y. Nishida, Phys. Rev. D 69, 094501 (2004) [hep-ph/0312371].

[6] H. Kluberg-Stern, A. Morel, and B. Petersson, Nucl. Phys. B 215 [FS7], 527 (1983).

[7] G. Faldt and B. Petersson, Nucl. Phys. B 265, 197 (1986).

[8] N. Bilic, K. Demeterfi, and B. Petersson, Nucl. Phys. B 377, 651 (1992); N. Bilic, F. Karsch, and K. Redlich, Phys. Rev. D 45, 3228 (1992); N. Bilic and J. Cleymans, Phys. Lett. B 355, 266 (1995).

[9] T. Jolicoeur, H. Kluberg-Stern, A. Morel, M. Lev, and B. Petersson, Nucl. Phys. B 235, 455 (1984).

[10] I. Ichinose, Phys. Lett. B135, 148 (1984); ibid. B147, 449 (1984); I. Ichinose, Nucl. Phys. B249, 715 (1985).

[11] K. Miura, T. Z. Nakano, and A. Ohnishi, Prog. Theor. Phys. 122, 1045 (2009) [arXiv:0806.3357 [nucl-th]]; K. Miura, T. Z. Nakano, A. Ohnishi, and N. Kawamoto, Phys. Rev. D 80, 074034 (2009) [arXiv:0907.4245 [hep-lat]].

[12] T. Z. Nakano, K. Miura, and A. Ohnishi, Prog. Theor. Phys. 123, 825 (2010) [arXiv:0911.3453 [hep-lat]]; T. Z. Nakano, K. Miura, and A. Ohnishi, Phys. Rev. D 83, 016014 (2011) [arXiv:1009.1518 [hep-lat]].

[13] F. Karsch and K. H. Mutter, Nucl. Phys. B 313, 541 (1989).

[14] P. de Forcrand and M. Fromm, Phys. Rev. Lett. 104, 112005 (2010) [arXiv:0907.1915 [hep-lat]]; W. Unger and P. de Forcrand, J. Phys. G 38, 124190 (2011) [arXiv:1107.1553 [hep-lat]]; M. Fromm, Lattice QCD at strong coupling: thermodynamics and nuclear physics, Ph.D. Thesis, ETH-19297, Eidgenössische Technische Hochschule ETH Zürich, (2010); M. Fromm and P. de Forcrand, PoS LATTICE2009, 193 (2009).

[15] P. de Forcrand, J. Langelage, O. Philipsen, and W. Unger, PoS LATTICE2013, 142 (2014) [arXiv:1312.0589 [hep-lat]]; W. Unger, Acta Phys. Polon. Supp. 7 No. 1, 127 (2014); Ph. de Forcrand, J. Langelage, O. Philipsen, and W. Unger, Phys. Rev. Lett. 113, 152002 (2014).

[16] A. Ohnishi, T. Ichihara, and T. Z. Nakano, PoS LATTICE2012, 088 (2012), T. Ichihara, T. Z. Nakano, and A. Ohnishi, PoS LATTICE2013, 143 (2014); T. Ichihara, A. Ohnishi, and T. Z. Nakano, Prog. Theor. Exp. Phys. 2014, 123D02 (2014) [arXiv:1401.4647 [hep-lat]].

[17] T. Ichihara, K. Morita, and A. Ohnishi, Prog. Theor. Exp. Phys. 2015, 113D01 (2015) [arXiv:1507.04527 [hep-lat]].

[18] F. Karsch, S. Ejiri, and K. Redlich, Nucl. Phys. A 774, 619 (2006) [hep-ph/0510126]; S. Ejiri, F. Karsch, and K. Redlich, Phys. Lett. B 633, 275 (2006); F. Karsch and K. Redlich, Phys. Lett. B 695, 136 (2011).

[19] R. -A. Tripolt, J. Braun, B. Klein, and B. -J. Schaefer, Phys. Rev. D 90, 054012 (2014); A. Bhattacharyya, R. Ray, S. Samanta, and S. Sur, Phys. Rev. C 91, 041901 (2015); A. Bhattacharyya, R. Ray, and S. Sur, Phys. Rev. D 91, 051501 (2015); X. Pan, L. Chen, X. S. Chen, and Y. Wu, Nucl. Phys. A 913, 206 (2013). 